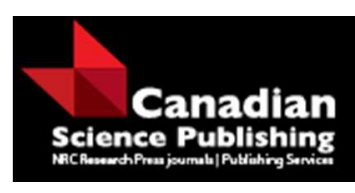

Canadian Journal of Physics

Revue canadienne de physique

\title{
Experimental Investigation of the Influence of Spatially Distributed Charges on the Inertial Mass of Moving Electrons as Predicted by Weber's Electrodynamics
}

\begin{tabular}{|r|l|}
\hline Journal: & Canadian Journal of Physics \\
\hline Manuscript ID & cjp-2017-0034.R1 \\
\hline Manuscript Type: & Article \\
\hline Date Submitted by the Author: & 22-Mar-2017 \\
\hline Complete List of Authors: & $\begin{array}{l}\text { Lőrincz, István; Technische Universitat Dresden, Institute of Aerospace } \\
\text { Engineering } \\
\text { Tajmar, M.; Technische Universität Dresden, Institute of Aerospace } \\
\text { Engineering }\end{array}$ \\
\hline Keyword: & $\begin{array}{l}\text { Weber's mass, intertial mass, potential dependent mass, relative motion, } \\
\text { spatial charge distribution }\end{array}$ \\
\hline $\begin{array}{r}\text { Is the invited manuscript for } \\
\text { consideration in a Special } \\
\text { Issue? : }\end{array}$ & N/A \\
\hline
\end{tabular}




\title{
Experimental Investigation of the Influence of Spatially Distributed Charges on the Inertial Mass of Moving Electrons as Predicted by Weber's Electrodynamics
}

\author{
István Lőrincz and Martin Tajmar
}

\begin{abstract}
It was shown (J. Phys. Soc. Jpn. 62, 1418 (1993).) that Weber's force law predicts an influence upon the apparent inertial mass of electrically charged particles in relative motion. The quantity of this influence, called Weber's mass, depends on the relative motion (both speed and acceleration) and the relative spatial distribution of the particles. A special case was analytically solved, in which the motion of a single point charge was considered that is surrounded by a static and fixed spherical surface with a homogeneous charge distribution. The first experimental test of the prediction showed positive results (Annal. Fond. Louis de Broglie, 24, 161 (1999).). Two reproductions were carried out, in which null results were reported together with an explanation for the previous positive result. It was suggested that a possible reason for the null result in the experimental setup could be that the charges could freely move on the surface. We set out to reproduce all previous experiments in order to get detailed insight into the problem, after which we designed a setup that would exclude the possibility of the moving charges on the surface. In all situations we could confirm the null results with a statistical precision of up to $0.009 \%$
\end{abstract}

Key words: Weber's mass, intertial mass, potential dependent mass, charged particles, relative motion, spatial charge distribution.

\section{Introduction}

An alternative mathematical formulation of the physics of electrodynamics was proposed by Weber [1], which would combine all the equations of classical electrodynamics, the Maxwell equations together with the Lorentz force law, into a single equation. As shown by Assis [1] all the classical properties of electromagnetism can be derived from the Weber equation. This equation reflects the results of all the observations of electromagnetism done up to the time of Weber. According to Weber the force between two electrically charged particles $q_{1}$ and $\mathrm{q}_{2}$ separated by a distance $\mathbf{r}_{21}$ is described by the equation:

$$
\vec{F}_{12}=\frac{q_{1} q_{2}}{4 \pi \varepsilon_{0}} \frac{\hat{r}_{21}}{r_{21}^{2}}\left(1-\frac{\dot{r}_{21}^{2}}{2 c^{2}}+\frac{r_{21} \ddot{r}_{21}}{c^{2}}\right)
$$

The proposed force, called the Weber force, is dependent on the relative velocity $\left(\dot{r}_{21}\right)$ and acceleration $\left(\ddot{r}_{21}\right)$ of the two interacting particles. Due to these two terms a new force component arises if an electrically charged particle is moving within the enclosed volume of a stationary, non-rotating charged spherical shell. First explicitly described and analytically derived by Assis [2], it was shown that the new force component in the ideal geometrical configuration is isotropic and is linearly dependent on the particle's acceleration relative to the center of the sphere. In this configuration, where a particle with the

István Lórincz ${ }^{\mathbf{1}}$ and Martin Tajmar. Institute of Aerospace Engineering, Technische Universität Dresden, 01307 Dresden, Germany

${ }^{1}$ Corresponding author (e-mail: istvan.lorincz@tu-dresden.de). charge $q$ is surrounded by a total charge $Q$ that is uniformly distributed over a spherical surface, the Weber force can be expressed as [2]:

[2] $\quad \vec{F}_{s}=\frac{q Q}{12 \pi \varepsilon_{0} c^{2} R} \vec{a}=\frac{q V}{3 c^{2}} \vec{a}$

where $\vec{a}$ is the acceleration of $q$ relative to the sphere's center, $R$ is the radius of the spherical shell, $\mathrm{c}$ is the speed of light in vacuum, $\varepsilon_{0}$ is the vacuum permittivity and $V=Q / 4 \pi \varepsilon_{0} R$ is the electrostatic potential inside the spherical shel referenced to infinity. If we now exert a force $\left(F_{e}\right)$ on the freely moving particle, having an inertial mass $m$, inside the sperical shell we obtain the following force equation by applying Newton's second law of motion $\left(v^{2} \ll c^{2}\right)$ :

$$
\begin{aligned}
& \vec{F}_{t}=\vec{F}_{e}+\vec{F}_{s}=\left(m-m_{w}\right) \vec{a} \\
& m_{w}=\frac{q Q}{12 \pi \varepsilon_{0} c^{2} R}=\frac{q V}{3 c^{2}}
\end{aligned}
$$

where $m_{w}$ is the parameter called the Weber mass.

For a more detailed and indepth description of Weber's electrodynamics the reader is referred to the works of Assis [2-4]. At this point it is worth noting that the subject is still actively being developed and thus both theoretical and experimental work has to be carried out simulatneously in order to achieve advancements. One such aspect that is important for this series of experiments is the covariance of the Weber equation [Eq. 1], which is still not firmly established. The previous demonstration for the covariance published in Assis's book [4] about relational mechanics is invalid because Lorentz transformations cannot be performed between frames of reference that possess relative acceleration. This raises significant doubts about some 
of the predictions of Weber's theory, which include Weber's mass [Eq. 4]. The difficult topic of covariance between accelerated frames in modern physics still needs to be properly developed and understood, thus experimental analyses would expedite this process at least for Weber's theory.

\subsection{The First Experiment}

Since this consequence is a crucial test for Weber's theory, experiments have been proposed $[1,2]$ and conducted by Mikhailov [5-7], Junginger et al. [8] and Little et al. [9].

Through the first experiment (schematic of our reproduction shown in figure 1), Mikahilov reported measurement results with the same order of magnitude as predicted by the theory (with applied approximations) [5]. In this experiment an oscillation circuit was constructed with the use of a neon glow lamp (ne-2) that produced discharges with a low frequency. Accidentally discovered and first described by Pearson and Anson [10] in 1921, the working principle of the oscillator circuit can be summarized as:

1. The capacitor $\mathrm{C} 1$ is charged up by the battery through the resistance $\mathrm{R} 1$

2. The voltage across $\mathrm{C} 1$ reaches the breakdown voltage of the glow lamp (approx. $90 \mathrm{~V}$ ) and the discharge is initiated within the glow lamp

3. $\mathrm{C} 1$ is discharged through the glow lamp and the resistor $\mathrm{R} 2$ until the lamp's extinction voltage is reached

A total period of the oscillation is composed of two main states:

a) Off-State: the time required for $\mathrm{C} 1$ to be charged up through R1.

b) On-State: the discharge time of $\mathrm{C} 1$ through the lamp and R2

An important property of glow discharge lamps is that the voltage necessary to initiate the discharge is usually higher than the voltage required to sustain the discharge (maintaining voltage). Hence in order to reach the Off-State after the first On-State a general condition of $\mathrm{R} 1 \gg \mathrm{R} 2$ needs to be satisfied (otherwise the maintaining current/voltage could be supplied through R1), while it is of interest to maximize the length of the On-State, which in turn is directly proportional to R2. Therefore we can conclude that the On-State will always be significantly shorter than $50 \%$ of the total period. An important factor which will be discussed later in this paper.

During the On-State a positive voltage impulse is generated over R2, which can be in turn directly read out on the output line by a counter to determine the frequency of the discharges. If we consider $\mathrm{C} 2$ as an ideal capacitor while fulfilling the condition $\mathrm{C} 1>\mathrm{C} 2$ then it has no influence on the frequency during the discharges. Although in reality there could be secondary effects which could indeed cause unwanted influences.

In the original setup, the electronic circuit (excluding the lamp) was placed inside a metal container (Faraday cage), while the glow lamp was placed in the center of a hollow glass sphere with an In-Ga alloy coating, sitting on top of the cage with an approx. $60 \mathrm{~cm}$ long neck (metal tube), which was charged up to $\pm 3000 \mathrm{~V}$. Mikhailov did not clearly specify which side of the glass had the coating.
Fig. 1. Mikhailov's setup reproduction schematic, showing the oscillator circuit powered by a $95 \mathrm{~V}$ battery (B), the NE-2 type neon lamp, the dielectric enclosure covered with metallic foil on the outside and the electrical connection between the high voltage source (enclosure) and the interior circuit's ground (K).The neck length is in this case approx. $6 \mathrm{~cm}$.

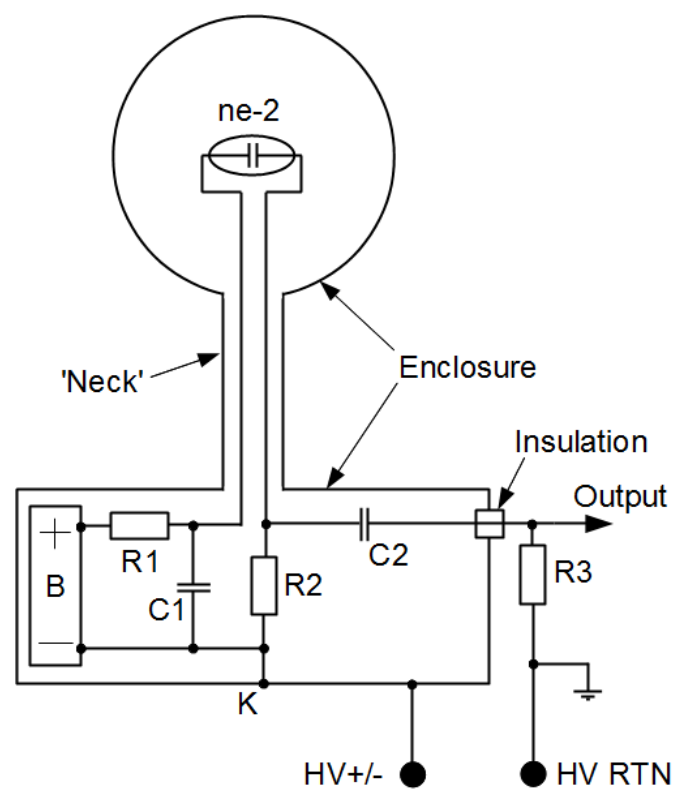

By assuming that the conditions within the lamp remain constant during the measurements, according to Mikhailov [5] a first order approximation of the circuit's differential resistance can be expressed as $R_{D}=k_{1} m_{0}$ where $\mathrm{k}_{1}$ is a proportionality constant and $m_{0}$ is the rest mass of the charge carriers. Thus the period of the discharge becomes $T_{0}=k_{2} R_{D}=k_{1} k_{2} m_{0}$ where $\mathrm{k}_{2}$ is a proportionality constant. This allows a definition of the discharge's period change in function of mass change, which in our case is assumed to be equal to $\mathrm{m}_{\mathrm{w}}$ :

$$
\frac{\Delta T}{T_{0}}=\frac{m_{w}}{m_{0}}=\frac{e V}{3 m_{0} c^{2}}
$$

where $\mathrm{e}$ is the elementary charge, $\mathrm{T}_{0}$ is the period of the oscillation at $0 \mathrm{~V}$ and $\Delta \mathrm{T}$ is the change of the period.

After this approximation a question still remains open, whether the expression for the differential resistance is applicable to both the electrically conductive wires (incl. the resistors) and the plasma discharge or there is a fundamental difference. We will see in the next chapter that indeed the latter is true and even in some special cases the approximation fails.

It will remain a mystery why Mikhailov chose this specific oscillator to measure tiny frequency changes, since it is associated with the first system in which chaotic behavior was observed [11].

\subsection{First Reproductions}

Mikhailov's report attracted some attention in the scientific community resulting in two replication attempts of the experiment $[8,9]$. In the case of Little et al. [9] the whole electronics including the discharge lamp was placed inside a relatively 
flat (compared to its radius) cylindrical metallic enclosure. The lamp within this cylindrical metallic enclosure had an arbitrary position and orientation, hence it can be stated that maintaining the isotropic property of the effect was disregarded during the replication. According to Assis [12] a cylindrical geometry does not provide an isotropic effect, although the maximum difference in the effect's magnitude due to the anisotropy is below one order of magnitude. Despite these differences the authors achieved to measure frequency changes in function of enclosure potential $( \pm 3000 \mathrm{~V})$. They suspected that the main cause for the dependency could be the coupling capacitor $\left(\mathrm{C}_{2}\right.$ in figure 1). To test this assumption four different types of capacitors with different capacitances were used to repeat the measurements. The trials confirmed the assumption, hence the conclusion was made that the results of Mikhailov were artifactual, due to the coupling capacitor.

In order to eliminate this artifact, the electrical coupling was replaced by an optical coupling. This led to the decrease of the previously mentioned dependence below the measurement's observable precision (approx. two orders of magnitude less than the theoretical prediction's value).

A similar experiment was carried out by Junginger et al. [8], who only used the optical coupling solution to measure the frequency of the neon glow lamp. A similar assumption was made in this experiment regarding the geometry of the enclosure, stating that the effect's order of magnitude remains the same if a cube is used instead of a sphere. They further introduce the idea that the charge carriers within the metallic conductors may not be affected the same way, from the point of view of Weber's mass, as they are within the discharge lamp. The duty cycle, the percentage of the total period during which the lamp is in the "On-State", was used as a correction factor for the effect's magnitude and hence implementing the previous assumption. Both assumptions are made without giving any explanation. We will attempt to give an explanation to this issue later.

The reported measurement results showed no correlation with the theoretical prediction with a relative error in the influence of enclosure potential on oscillation frequency of $1.4 \times 10^{-5}$, which is two orders of magnitude less than their expected magnitude of $1.4 \times 10^{-3}$.

In this replication attempt the enclosure was made of an unspecified material which was covered with a metallic foil. This difference becomes potentially significant in case if we consider the dynamic interactions between the charge carriers on the surface of the enclosure and those within. Based on these considerations it was suggested by Assis $[2,12]$ that the theoretically predicted Weber mass by Mikhailov [2] could be decreased due to image charges and/or induced currents on the sphere surrounding the discharge lamp.

\section{Experiment Setups and Results}

The classical electrostatic problem of a point charge at a distance from the center of a perfectly conducting sphere was solved by Lord Kelvin (William Thomson) in 1845 [13]. Since the problem is symmetric, we can assume that the real charge is within the sphere and the image charge is on the outside. The schematic of the problem can be seen in figure 2. By applying Lord Kelvin's method of image charges we can only infer the
Fig. 2. The schematic for Lord Kelvin's application of the electrostatic images method for a point charge (q) at a distance (p) from the center of a perfectly conducting grounded hollow sphere $(S)$ with a radius $R$

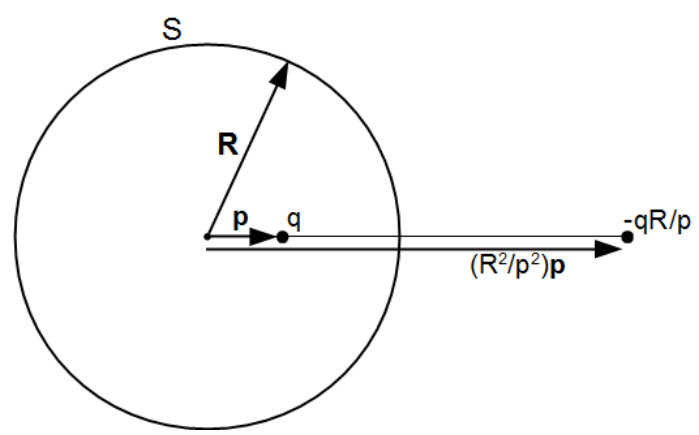

change of the total charge on the sphere's surface. We can quickly draw a final conclusion, without making any calculations, if we consider the fact that the discharge is composed of a neutral plasma. Meaning that the distance between the positive and the negative charge carriers is negligible compared to the overall geometry leading to a net total surface charge change of zero.

We face a totally different situation if we want to analyze the possibility of induced surface currents and how it affects Weber's mass. In this case an analytical approach can not be applied since the problem is too complex. Therefore only two options remain:

a) Experimental evaluation, where the problem is excluded or minimized

b) Analysis through numerical simulation

In order to obtain an answer we chose the former, so we implemented a set of experiments that would first reproduce the previous measurements and then build a setup where the possibility of induced currents is minimized. We performed three types of experiments, in which we tried to measure an influence of spatially distributed electric charges on the inertial mass of moving electrons:

I. Reproduction of Mikhailov's Setup

(a) Electrical signal coupling

(b) Optical signal coupling

\section{Electric Charge Enclosure with Electrets}

\subsection{Reproduction of Mikhailov's Setup}

As a first step a reproduction of the Mikhailov setup was considered necessary as a baseline experiment, while noting that we chose to have a slightly different blinking frequency of the glow lamp. Our circuit produced a frequency of about $35 \mathrm{~Hz}$ while the frequency of Mikhailov's setup was $138 \mathrm{~Hz}$. We observed that by lowering the frequency the stability increased, meaning that even though we took the average frequency during a specified period of time the standard deviation got smaller. Since the blinking frequency had a considerable 
Fig. 3. Mikhailov's setup reproduction results compared to the theoretical prediction ( $35 \mathrm{~Hz}$ nominal frequency)

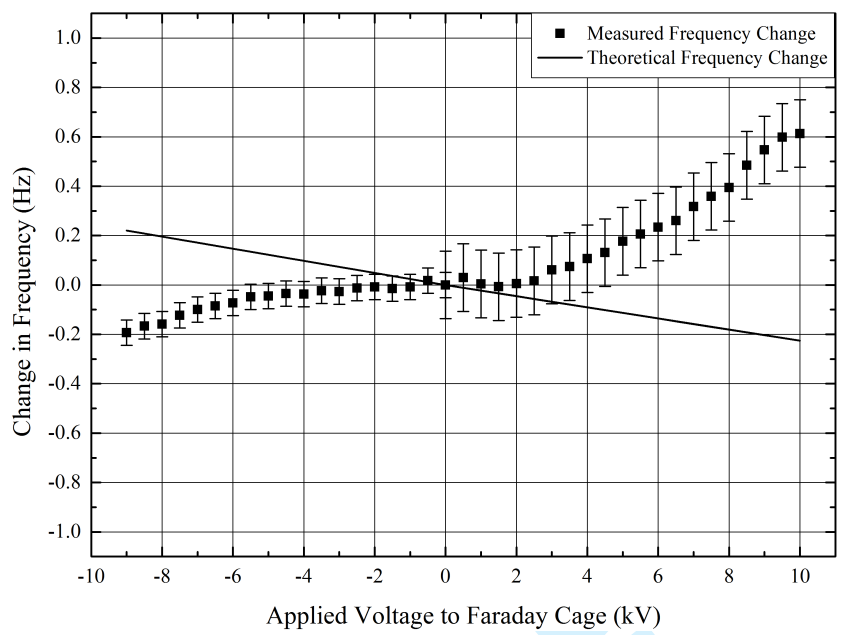

drift in each setup, a standard measurement approach was applied, in which the voltage of the sphere was varied periodically from 0 to the target value and back to 0 . This way the drift could be removed through simple signal post-processing. The box containing the driving circuit was made out of Styrofoam on top of which was the glass sphere placed with a radius of approx. $5 \mathrm{~cm}$ and a neck length of about $6 \mathrm{~cm}$. The outside of the whole assembly was completely and tightly covered by aluminum foil, instead of the metallic coating.

An important detail of the setup is the connection between the oscillation circuit's ground and the Faraday cage, which in turn is connected to the high voltage supply. It was claimed by Mikhailov [5] that in case this connection is not present the sought after effect was not measurable. Thus we tried both variations where the battery ground was disconnected and then connected to the Faraday cage, but we observed no difference between the two configurations. The result from the first setup can be seen in figure 3, where the ground connection was applied. The difference between the uncertainty bars originates from the high voltage supply, which introduced different noise levels in our DAQ system for the two polarities.

A change in frequency can be observed, which is within the same order of magnitude as predicted by the theory, however instead of the theoretical linear function with a negative slope we obtained a positive cubic function. As previously shown by Little et al. [9], the frequency change was expected due to the coupling capacitor. No further investigation was made since the optical coupling was the next step, through which we expected the effect to disappear.

\subsection{Mikhailov's Setup with Optical Coupling}

In order to exclude every possible influence caused by the coupling capacitor, another solution to read out the blinking frequency was implemented. An optical coupling is ideal in this case since the blinking frequency can be read out directly without influencing the electrical circuit's operation. An optical fiber was fixed close to the lamp's surface, which transmitted the signal to a counter through a photo-diode connected to
Fig. 4. Tunable optical signal conditioning circuit

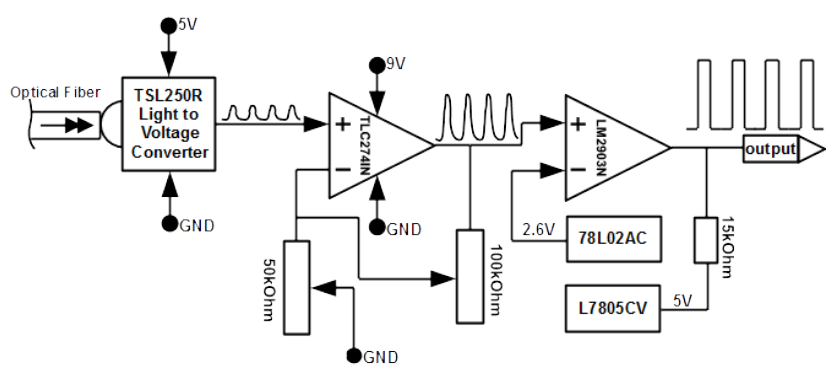

Fig. 5. Measurement result with optical coupling - direct measurement of time between each consecutive pulse $(35 \mathrm{~Hz}$ nominal frequency)

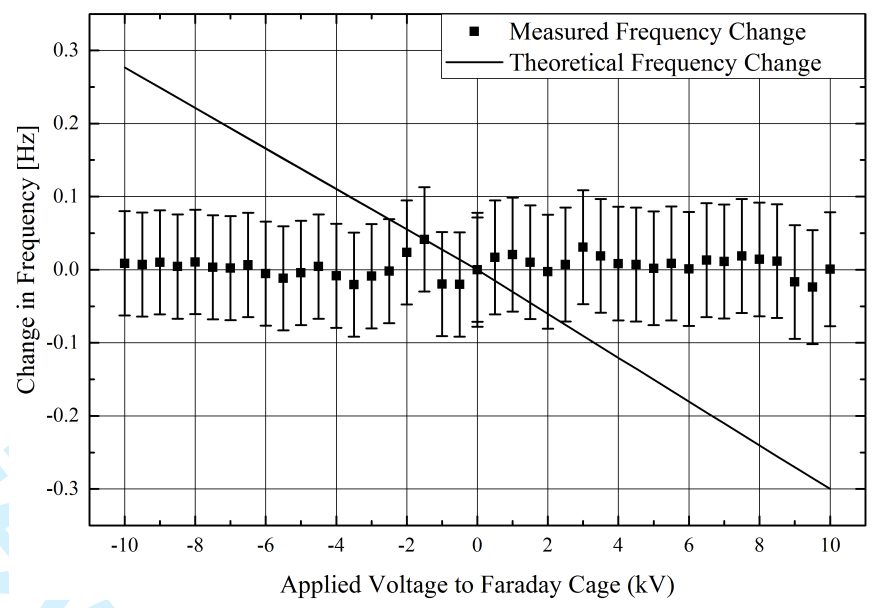

an adjustable amplification and pulse shaping circuit (figure 4).

With this setup two types of measurements were performed. In the first set of measurements each pulse length was measured during a specified period (in the order of seconds), which was in turn averaged. This approach gave a greater statistical insight into the data (at the cost of precision) than the second set of measurements (the approach of Junginger et al.) where the number of pulses was measured during a much longer period (in the order of minutes). Both measurements gave a negative result within a statistical precision of up to $0.009 \%$, which can be seen in figures 5 and 6 for the first and the second measurement approach respectively.

It has to be noted that for the second measurement set we aimed at the reproduction of the Junginger et al. setup, for which we had to increase the blinking frequency to approximately $700 \mathrm{~Hz}$ by simply adjusting the RC circuit.

For the theoretical prediction in the counting measurements the assumption was made that the mass change only occurs within the discharge, leaving the rest of the electrical circuit unchanged. This has a large impact upon the amplitude of the effect, since the actual discharge takes up only $20 \%$ (the lamp's duty cycle) of the total period. Further taking into consideration that the discharge doesn't produce an instantaneous, ideal and constantly accelerating beam of electrons in free space, but is a result of a succession of various physical processes, the 
Fig. 6. Measurement result with optical coupling - counts during $100 \mathrm{~s}$ with $\pm 16 \mathrm{kV}$ enclosure bias compared to theoretical predictions $(700 \mathrm{~Hz}$ nominal frequency)

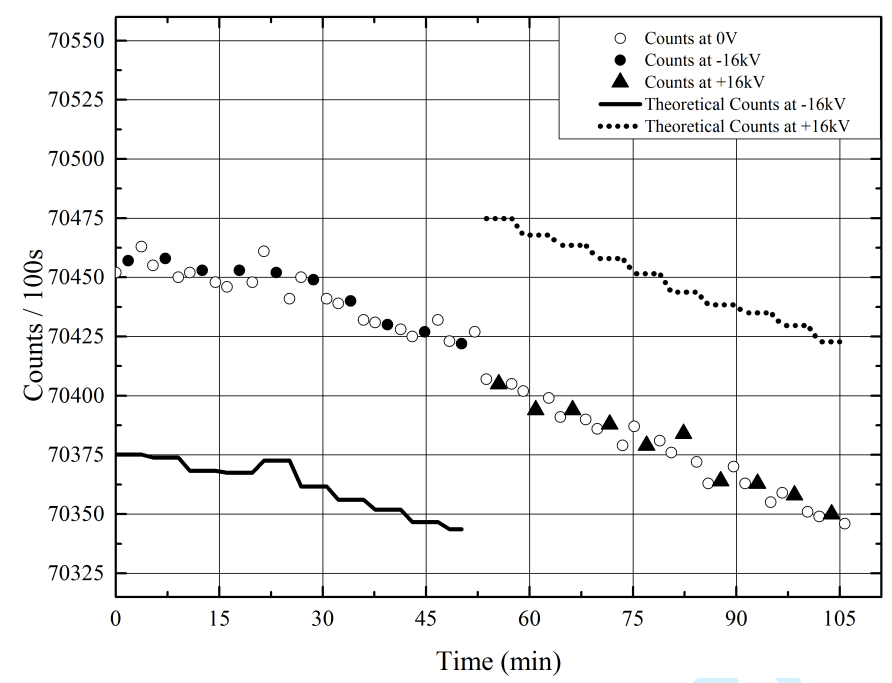

amplitude of the assumed effect would further decrease. There are numerous other unknown influences that definetly change the effect, just to name a few:

1. Townsend discharge

2. Change in breakdown voltage

3. Particle collision crossections (e.g. charge exchange, momentum transfer, ionization, etc.)

\section{Sputtering}

In order to take these into account a detailed study would be required to define an accurate correction factor, which is beyond the scope of this paper. In our case it is enough to find a first order estimate expressed through a correction factor equal to the lamp's duty cycle. Thus the theoretical change in the period becomes:

$$
\Delta T=\frac{e U}{3 c^{2} m_{0}} T_{0} D_{L}
$$

where $\mathrm{D}_{\mathrm{L}}$ is the lamp's duty cycle.

\subsection{Electret Setup}

As it was mentioned previously a possible cause for the negative results could be the effect of image charges on the surface of the enclosure. In order to exclude this possibility and to get a step closer to the ideal case, where the spherical shell is composed of spatially fixed charges, a new setup was designed. The easiest way to approximate this situation is by replacing the metallic enclosure with an electret.

Electrets are dielectric materials that have a quasi-permanent electric charge. In these materials the electric charge carriers cannot move like in metals, hence the charge distribution can be approximated as static during a relatively long period of
Fig. 7. Charge deposition on a dielectric surface

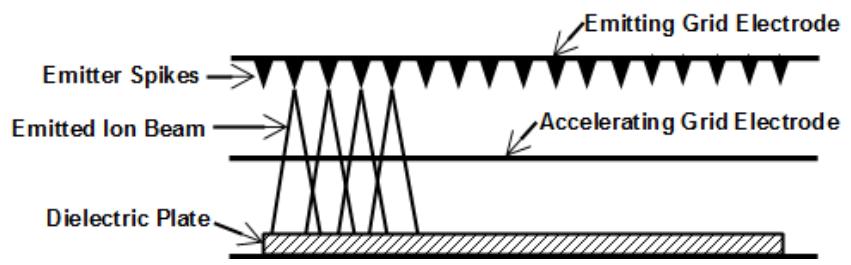

time (in the order of hours) even in the presence of an outside electromagnetic field. The only drawback of this approach is that it is quite difficult to produce electrets, which have a homogeneous surface charge distribution, especially when the surface is spherical. Thus a compromise has been made to switch the spherical geometry to a cube. This way only six dielectric plates had to be charged up, which could be made with a relatively accurate repeatability. This change of course also had an impact on the effect itself, but according to Assis it would only eliminate the property of isotropy. The effect's magnitude would reach maximum when two of the cube's surfaces would be perpendicular to the average velocity vector of the charges within the discharge.

Since the electrets had to be produced on site, a charge deposition solution was necessary. We chose to produce the electrets by using the well-known corona discharge method. The schematic of our method can be seen in figure 7 .

The evident goal was to achieve the maximum surface charge density with the available high voltage supply, which was capable of producing $\pm 20 \mathrm{kV}$ on two outputs. Thus the total available potential difference was $40 \mathrm{kV}$. There are two further parameters that had to be optimized in this setup, namely the distance between the emitting and accelerating grid, and the emitted current. This was realized experimentally by varying the electrode separation. Evidently there is still the distance between the dielectric plate and the accelerating electrode, which has an optimum distance. This was also determined experimentally by varying the distance and measuring the resulting surface potential on the dielectric plate after a specified amount of charge deposition time. To determine the surface potential of the plates, an electrostatic field meter (Monroe-electronics Model 255) was used with a fixed distance of 1 inch from the measured surface. After the geometric parameters have been defined, they were kept constant during the experiment.

Numerous materials were tested that would be viable for the dielectric box, in order to determine which is best suited for the experiment. The most important factor was the discharge time (or the rate at which a plate would loose its electric charge), since the charging of one plate required between 14 to $15 \mathrm{~min}$ utes and about 50 minutes were necessary to complete the measurements. The best material turned out to be PTFE plates $(19.5 \mathrm{~cm} \times 19.5 \mathrm{~cm})$, which could hold an approximately constant charge for up to 2.5 hours after a settling period of about 10 minutes. With this solution a maximum electrostatic field magnitude of $-8.3 \mathrm{kV} /$ in at 1 inch distance from the surface's center could be reached. This value decreased by up to $15 \%$ within the first 15 minutes, after which a steady value (within $\pm 2 \%$ ) was held for a few hours. However the distribution on the surface could not be made homogeneous, it decreased towards the edges of the plates by up to $28 \%$ of the maximum 
Fig. 8. Schematic of the experiment with the electret box. The discharge lamp was positioned inside the box such that the charge carriers' velocity vector would be perpendicular to the walls of the box, as illustrated by the "glow-discharge direction"

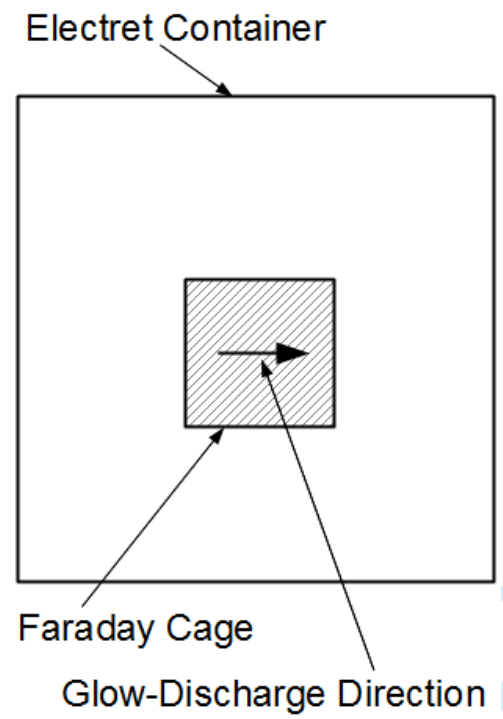

value at the center. This would further introduce complications, since the equivalent homogeneous charge distribution geometry for each plate would be a convex surface from the point of view of the center of the box (figure 8) where the lamp was placed. In order to obtain an approximation for the equivalent surface potential of a charged conducting plate, we can consider an infinitesimally thin conducting disk with the same surface area as one of the PTFE plates and apply the following equations:

$$
V_{e q}=\frac{Q_{e q}}{C_{e q}}=\frac{\sigma_{e q} \pi r^{2}}{8 \varepsilon_{0} r}=\frac{E \pi r}{8}
$$

$$
\sigma_{e q}=E \varepsilon_{0}
$$

where $\mathrm{V}_{\mathrm{eq}}$ is the equivalent potential of the conducting disk, $\mathrm{Q}_{\mathrm{eq}}$ is the total charge, $\mathrm{C}_{\mathrm{eq}}$ is the self-capacitance of an infinitesimally thin conducting disk, $\sigma_{\text {eq }}$ is the surface charge density, $\mathrm{r}$ is the radius of the disk, $\varepsilon_{0}$ is the vacuum permittivity and $E$ is the measured electrostatic field strength. This way we can use the same theoretical prediction as in the previous experiments. In our case the equivalent potential of the electrets were approximately $-15 \mathrm{kV}$.

In this situation we have to consider also the fact that the electrostatic field strength inside the box is not zero. In order to exclude the effect of the field, the driving circuit together with the lamp was placed inside a Faraday cage. This would not cancel out the effect according to Assis and Mikhailov [6], who also conducted an experiment where he used a Faraday cage inside the charged sphere and claimed to have measured the same effect. The assembled electret box can be seen in figure 9 .

The measurements were done again by alternating the lamp's surrounding potential from $0 \mathrm{~V}$ to $-15 \mathrm{kV}$. This was done by placing the driving circuit together with the lamp outside of
Fig. 9. Assembled box of separate PTFE plates after charge deposition

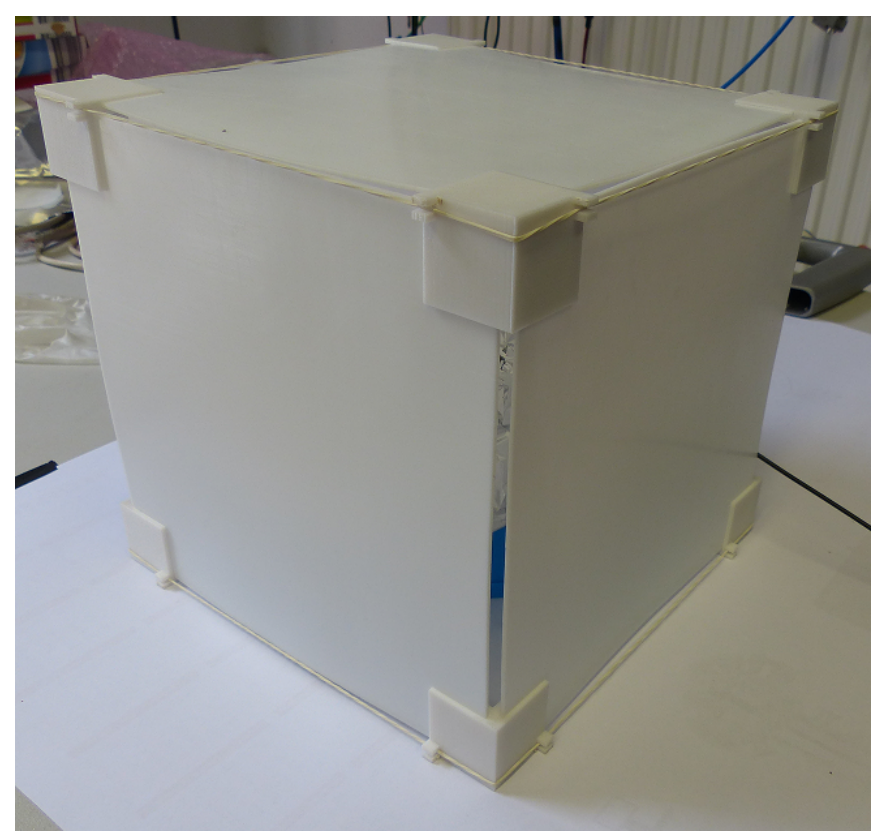

the box, then placing them back inside. The result of the measurements can be seen in figure 10 .

The dash-dotted line represents the number of counts according to the theory including our correction factor for the case where the discharge lamp is placed inside the electret box. The measurement shows no difference in the number of counts within our precision between the two cases of $0 \mathrm{~V}$ and $-15 \mathrm{kV}$ surrounding equivalent surface potential.

\section{Conclusions and Discussions}

Four types of measurements were carried out in order to test Weber's theory of electrodynamics that predicts an inertial mass change of freely moving electrical charges within an electrostatically charged hollow sphere. In all of the measurements the frequency of a glow discharge neon lamp oscillator was measured, which was surrounded by electrical charges. We successfully reproduced the previously conducted experiments, which had contradictory results. We first reproduced the experiment of Mikhailov and obtained an effect with a similar order of magnitude change in frequency, although with an opposite slope. We identified the cause of the effect by changing the method of measuring the discharge rate from an active electrical coupling to a passive optical coupling. Through this modification the theory remained unchanged and we could successfully confirm the negative results previously published by Junginger et al. and Little et al. In addition we tested the assumption that the charges inside the discharge lamp are affecting the charges on the surface of the conducting sphere, thus diminishing the assumed effect. This was achieved by depositing electric charges on PTFE plates, which would act as quasistatic and fixed charge holders. Hence a new spatial charge distribution geometry has been created for the enclosure that is 
Fig. 10. Measurement result with the electret box - counts during $100 \mathrm{~s}$ with approximately $-15 \mathrm{kV}$ enclosure bias compared to the theoretical prediction

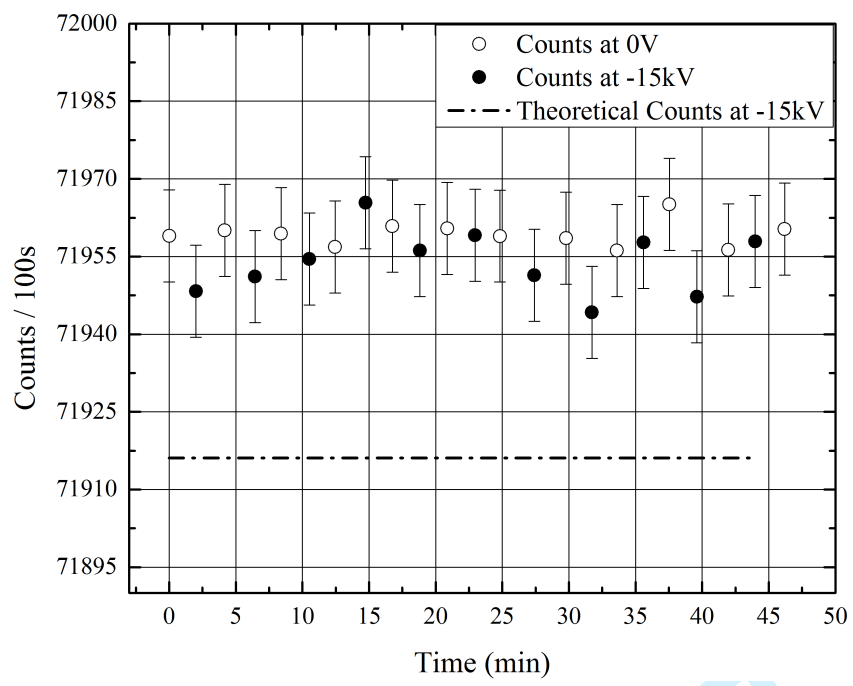

a closer approximation to the ideal case from which the analytic solution was deduced. Even though the major differences between theory and experiment have been considered and expressed with correction factors, a negative result has been obtained within a statistical precision of up to $0.009 \%$.

Although all these measurements have produced a negative result, the main question is still not answered with a satisfactory certainty. A very important factor has not been considered within these experiments, namely the electrical composition of the discharge. It is clear that the current within a low pressure discharge lamp is conducted through plasma, which contains the same number of positive and negative charges. Hence the question arises what is the actual quantitative impact of the discharge composition upon the assumed effect's magnitude. In order to answer this question, detailed numerical simulations have to be carried out in which all the transient processes are considered during a discharge. Another approach would be to completely get rid of the positive charges and generate a discharge or a beam consisting only of electrons. Even in this situation one should consider the charge distribution within the electron beam, because transient dynamical effects could potentially decrease the observable effect's magnitude. A much better measurement would be to use a narrow electron beam upon which a constant magnetic field is applied. In this situation the electron beam is deviated with a certain radius that is linearly dependent on the mass of the electrons. A precise measurement of the trajectory of the electrons could give a definite and certain answer to the question whether Weber's electrodynamics is correct. This problem was most likely also recognized by Mikhailov [7], who came up with an experiment in which he measured the frequency of a Barkhausen-Kurz oscillator that uses only electrons to produce very high frequencies. The frequency within such an oscillator has the same linear dependency of the electron's inertial mass as in the experiment previously proposed. This experiment was recently reproduced at TU Dresden [14], during which we discovered that the setup of Mikhailov did not produce Barkhausen-Kurz oscillations. In order to obtain this special operation mode of some electron tube types, we modified the circuit until we could confirm that indeed a Barkhausen-Kurz oscillation is produced, after which we performed the measurements. The results were again inconclusive because of the same reasons as with the glow discharge. It is easy to predict the behaviour of a single oscillating electron while its mass is being changed. The situation becomes almost impossible to predict without detailed numerical simulations if we consider an oscillating electron cloud having a normal distribution in phase space, which is the case for the Barkhausen-Kurz oscillation.

\section{Acknowledgements}

The authors would like to thank A.K.T. Assis for the encouragement to conduct the experiments and for providing helpful advice during the preparation of the measurements.

\section{References}

1. A. K. T. Assis, "Weber's Electrodynamics", Kluwer Academic Publishers ISBN 0-7923-3137-0 (1994)

2. A. K. T. Assis. J. Phys. Soc. Jpn. 62, 1418 (1993)

3. A. K. T. Assis and J. J. Caluzi,'A limitation of Weber's law", Physics Letters A, Vol. 160, pp. 25-30 (1991)

4. A. K. T. Assis, "Relational Mechanics and implementation of Mach's Principle with Weber's Gravitational Force”, ISBN 9780-9920456-3-0 (2014)

5. V. F. Mikhailov, Annal. Fond. Louis de Broglie, 24, 161 (1999)

6. V.F. Mikhailov, Annal. Fond. Louis de Broglie, 28 (2003)

7. V.F. Mikhailov, Annal. Fond. Louis de Broglie, 26 (2001)

8. J. E. Junginger, Z. D. Popovic, Can. J. Phys. 82: 731735 (2004)

9. S. Little, H. Puthoff, M. Ibison, "Investigation of Weber's Electrodynamics", (2001)

10. S. O. Pearson and H. St. G. Anson, Proc. Phys. Soc. London 34 175, (1921)

11. B. van der Pol, J. van der Mark, Nature (MacMillan) 120 (3019): 363364, (1927)

12. Personal correspondence with A.K.T. Assis at TU Dresden, (2014)

13. W. Thomson (Kelvin), "Extrait d'une lettre de M. William Thomson à M. Liouville." originally in Journal de Mathematiques Pures et Appliquées, Vol. 10, pp. 364, (1845)

14. Tajmar, M., "Revolutionary Propulsion Research at TU Dresden", Proceedings of the Advanced Propulsion Workshop, Estes Park, 20-22 Sept (2016) 